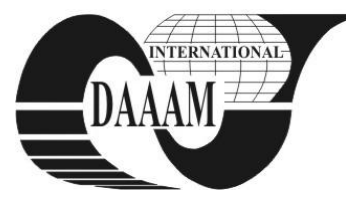

Annals of DAAAM for 2011 \& Proceedings of the 22nd International DAAAM Symposium, Volume 22, No. 1, ISSN 1726-9679 ISBN 978-3-901509-83-4, Editor B. Katalinic, Published by DAAAM International, Vienna, Austria, EU, 2011

\title{
ANALYZING THE FLUID MOTION THROUGH NETWORK PROFILES USING THE BOUNDARY ELEMENT METHOD
}

\author{
KOVACS, A[dalbert] \& KOVACS, L[aura]
}

\begin{abstract}
In this paper we present a calculus algorithm for the study of the compressible fluid's stationary motion through profile grids, on an axial-symmetric flow-surface, in variable thickness of stratum. We show the applicability of the boundary element method (BEM) with real values, and the possibility of solving the integral equation of the velocity potential by using the successive approximation method w.r.t. the fluid's density and the thickness variation of fluid stratum. In our approach the derivatives of the velocity potential are calculated by employing the Lagrange interpolation formula through five points.
\end{abstract}

Key words: boundary element method, hydrodynamics, Lagrange interpolation, compressible fluid

\section{INTRODUCTION}

Based on the boundary element method (BEM) with real values, in (Hromadka II., T.V. 1984) a variant of the BEMmethod in complex variables is presented, called the CVBEM method. While the mathematical framework of (Hromadka II., T.V. 1984) is in general very expressive, the introduced CVBEM method can only be applied for solving boundary value problems of bi-dimensional Laplace equations. As hydrodynamical problems not always can be modelled using such equations, the restrictions of (Hromadka II., T.V. 1984) impose severe limitations, for example, in the study of the compressible fluid's motion through profile grids.

In (Kovacs, A. 2005) the study of the compressible fluid's motion through profile grids is addressed using the integral equation method. According to (Kovacs, A. 2005), the complex potential of the fluid's motion is a p-analytic function. The Cauchy integral formula for analytic functions cannot thus be used, and hence the CVBEM method given in (Hromadka II., T.V., 1984) cannot be either applied.

In this paper we address the limitations of (Hromadka II., T.V. 1984), and describe adjustments of the BEM and CVBEM methods for the study of the compressible fluid's motion. For doing so, we deploy the fundamental equations of the compressible fluid's motion, and solve the resulting integral equations. For doing so, we use BEM with real values in conjunction with a successive iteration method w.r.t. the fluid's density (Section 3). Our method yields, in particular, an algorithmic approach for deriving the fluid's complex velocity (Section 4).

\section{PRELIMINARIES}

The fundamental equations describing the compressible fluid's motion on an axial-symmetric flow-surface, in variable thickness of stratum $h$, are given in (1).

$$
\begin{aligned}
& \bar{w}(z)= \bar{V}_{m}+\int_{L_{0}} H(z, \zeta) \bar{w}(\zeta) d \zeta+i \iint_{D_{0^{*}}} H(z, \zeta) g(\zeta) d \xi d \eta \\
& F(z)=\bar{V}_{m} \cdot z+\Gamma \cdot G\left(z, \zeta_{A}\right)+\int_{L_{0}} H(z, \zeta) F(\zeta) d \zeta+ \\
& \quad i \iint_{D_{0^{*}}} G(z, \zeta) \bar{q}(\zeta) d \xi d \eta
\end{aligned}
$$

where $\bar{V}_{m}=\frac{\bar{V}_{1 \infty}+\bar{V}_{2 \infty}}{2}$ is the asymptotic mean velocity; $\overline{\mathrm{w}}(\mathrm{z})=\mathrm{v}_{\mathrm{x}}-\mathrm{iv}_{\mathrm{y}}$ is the complex conjugate velocity; and $F(z)=\varphi+i \psi$ denotes the complex potential, where $\varphi$ is the velocity potential and $\psi$ is the flow rate function. The functional components of (1) are defined below:

$$
\begin{aligned}
& H(z, \zeta)=\frac{1}{2 t i} \operatorname{ctg} \frac{\pi}{t}(z-\zeta), \quad G(z, \zeta)=\frac{1}{2 \pi i} \ln \sin \frac{\pi}{t}(z-\zeta) \\
& \hat{q}(\zeta)=2 \frac{\partial \bar{w}}{\partial \bar{\zeta}}=-\left[v_{x} \frac{\partial \ln p^{*}}{\partial \xi}-v_{y} \frac{\partial \ln p^{*}}{\partial \eta}\right], \\
& p^{*}=\frac{\varsigma \cdot h}{\varsigma_{0}}
\end{aligned}
$$

where $t$ is the grid step; $\Gamma$ is the circulation around the base profile $L_{O}$; and $D_{0 *}^{-}$denotes the bounded simple convex domain:

$$
D_{0^{*}}^{-}:\left[-\frac{t}{2}\left\langle\xi \left\langle\frac{t}{2},-\left(t+\frac{l}{2}\right)\left\langle\eta\left\langle\left(t+\frac{l}{2}\right)\right]\right.\right.\right.\right.
$$

In (3), $l$ is the projection of $L_{0}$ on the Oy axis. Based on (Kovacs, A. 1999), the complex conjugate velocity $\bar{w}(z)$ is in practice determined by an a priori fixed iteration formula.

\section{SOLVING THE INTEGRAL EQUATION OF THE VELOCITY POTENTIAL}

A rigorous analysis of (1) requieres a detailed study of the complex velocity, the flow rate function and the velocity potential. In what follows, we discuss our approach for computing the value of these functions.

In our work we rely on the results of (Kovacs, A. \& Kovacs, L. 2003) and deploy the BEM method for solving (1) in real variables. To this end, we consider the integral equation of the complex potential $\mathrm{F}(\mathrm{z})=\varphi+i \psi$, and transform it into an integral equation with real variables. That is, we build the integral equation of the velocity potential $\varphi(x, y)$. For doing so, we note that the velocity potential $\varphi(\mathrm{s})$, with $s \in L_{0}$, is the solution of the integral equation (4):

$$
\varphi(s)+\int_{L_{0}} \varphi(\sigma) \frac{d M(s, \sigma)}{d \sigma} d \sigma=b(s)+\iint_{D_{0^{*}}} \widehat{q}(\sigma) N(s, \sigma) d \xi d \eta
$$

where:

$$
\begin{aligned}
& b(s)=2\left(x_{0} V_{m x}+y_{0} V_{m y}\right)+\Gamma M\left(s, \sigma_{A}\right)+\int_{L_{0}}[\psi(s)-\psi(\sigma)] \frac{d N}{d \sigma} d \sigma \\
& M\left(z_{0}, \zeta\right)=\frac{1}{\pi} \operatorname{arctg} \frac{\operatorname{th} \frac{\pi}{t}\left(\eta-y_{0}\right)}{\operatorname{tg} \frac{\pi}{t}\left(\xi-x_{0}\right)} \\
& N\left(z_{0}, \zeta\right)=\frac{1}{\pi} \ln \sqrt{\frac{1}{2}\left[\operatorname{ch} \frac{2 \pi}{t}\left(\eta-y_{0}\right)-\cos \frac{2 \pi}{t}\left(\xi-x_{0}\right)\right]}
\end{aligned}
$$


Note that equation (4) is an integral-differential equation. Hence, based (Kovacs, A. \& Kovacs, L. 2003), it can be solved by the successive approximation (iteration) method w.r.t. the parameter $\mathrm{p}^{*}=\frac{\varsigma}{\varsigma_{0}} \mathrm{~h}$. We recall that for isentropic processes, using the Bernoulli-equation, we have:

$$
\zeta=\varsigma_{0}\left(1-\frac{\gamma-1}{2} \frac{\mathrm{v}^{2}}{\mathrm{c}_{0}^{2}}\right)^{\frac{1}{\gamma-1}}, \mathrm{v}^{2}=\mathrm{v}_{\tau}^{2}+\mathrm{v}_{\mathrm{n}}^{2},
$$

where $\gamma$ is the adiabatic constant; and $\mathrm{c}_{0}$ is the sound velocity in the zero velocity point. In each step of the successive approximation method, a Fredholme integral of order II is obtained, for which, using the supraposition rule of flow potentials, we seek solutions of the form:

$$
\begin{aligned}
& \varphi^{(\mathrm{n})}=\varphi_{1}^{(\mathrm{n})} \mathrm{V}_{\mathrm{mx}}+\varphi_{2}^{(\mathrm{n})} \mathrm{V}_{\mathrm{my}}+\varphi_{3}^{(\mathrm{n})} \Gamma+\varphi_{4}^{(\mathrm{n})} \mathrm{u}_{0} \\
& \mathrm{u}_{0}=\omega \mathrm{R}_{0}, \quad \mathrm{n}=1,2, \ldots
\end{aligned}
$$

Solving each integral equation reduces to solving a system of linear algebraic equations of the form:

$$
\varphi_{k i}^{I}+\sum_{j=1}^{2 n-1} \varphi_{k j}^{I} \Delta M_{i j}=b_{k i}, i=1,3, \ldots, 2 n-1, k=1,2,3,4
$$

where:

$$
\begin{aligned}
& b_{1 i}^{I i}=2 x_{i}, \quad b_{2 i}^{I}=2 y_{i}, \quad b_{3 i}^{I}=M_{i, A} \\
& b_{4 i}^{I}=\sum_{j=1}^{2 n-1} \Delta \psi_{i, j}^{I}\left(\frac{d N}{d \sigma}\right)_{i, j} \Delta \sigma_{j} \\
& \Delta \psi_{i, j}^{I}=\psi_{i}^{I}-\psi_{j}^{I}, \quad \Delta \sigma=\sigma_{j+1}-\sigma_{j-1}
\end{aligned}
$$

By solving the algebraic system (7), we obtain $\varphi_{k i}^{I}$ in $n$ distinct points from the boundary of $L_{0}$. Finally, from equations (6), $\varphi_{i}^{I}$ is inferred in each point of the boundary's division. The complex velocity $v(s)$ is further calculated based on the following equations:

$$
\begin{aligned}
& \mathrm{v}^{2}(\mathrm{~s})=\mathrm{v}_{\tau}^{2}(\mathrm{~s})+\mathrm{v}_{\mathrm{n}}^{2}(\mathrm{~s}), \\
& \mathrm{v}_{\tau}(\mathrm{s})=\frac{\mathrm{d} \varphi}{\mathrm{ds}}, \quad \mathrm{v}_{\mathrm{n}}(\mathrm{s})=\frac{1}{\mathrm{p}^{*}} \frac{\mathrm{d} \psi}{\mathrm{ds}}
\end{aligned}
$$

The flow rate function is determined from the following boundary condition:

$$
\psi(s)=u_{0} \cdot \int_{0}^{s} p^{*}(s)\left(\frac{R}{R_{0}}\right) d s, u_{0}=\omega R_{0}
$$

where $\omega$ is the angular rotation velocity of the profile grid; $R_{0}$ defines the origin of the axis system related to the turbine's axis; and $R$ is the radius of the circular motion of the current point. Using numerical integration methods in conjunction with the successive approximation method, from (10) we get $\psi_{\mathrm{j}}^{(\mathrm{n})}$, with $j=1,2, \ldots$, in $n$ distinct points of the base profile $L_{0}$.

We finally calculate the complex velocity values $w_{i}=\left|\overline{\mathrm{W}}_{\mathrm{i}}\right|$, $\mathrm{w}_{\mathrm{i}}^{2}=\mathrm{v}_{\tau_{\mathrm{i}}}^{2}+\mathrm{v}_{\mathrm{n}_{\mathrm{i}}}^{2}$, for $i=1,3, \ldots, 2 m-1$, by applying the Lagrange interpolation formula through five points over the velocity potential $\varphi(s)$ and flow rate function $\psi(s)$. We refer to (Kovacs, A. \& Kovacs, L. 2006) for more details.

Let us note that the efficiency of our method crucially depends on the chosen division over the boundary of $L_{0}$. Experiments show that our method scales well when the division is set to have at least 60 points. Theoretical investigation for understanding how to choose the best division is left for future work.

\section{THE CALCULUS ALGORITHM OF THE FLUID'S COMPLEX VELOCITY}

We have now all ingredients to formulate our algorithm for calculating the fluid's complex velocity, as follows.

1. We are given the entering values of $p_{1}, v_{100}, \alpha_{1}$ into the profile grids; the asymptotic mean velocity $\vec{V}_{m}$; the installation angle $\lambda$; the number of profiles $n$; the density $\varsigma_{0}$; and the sound velocity $c_{0}$ corresponding to the null-velocity point. The functions $h(\sigma)$ and $\mathrm{R} / \mathrm{R}_{0}(\sigma)$ are given by their value tables.

2. Conform to the chosen division, the coordinates $\sigma_{\mathrm{i}}\left(\xi_{\mathrm{i}}, \eta_{\mathrm{i}}\right)$, with $i=1,3,5, \ldots, 2 n-1$, are determined.

3. Using equation (5), the values of $\Delta M_{i, j},\left(\frac{d N}{d \sigma}\right)_{i, j}, I$ with

$j=1,3,5, \ldots, 2 n-1$, are calculated.

4. Using the trapezoid method, $\psi_{i}^{I}$ is inferred from the integral equation (11). By (9), $\mathrm{b}_{\mathrm{ki}}^{\mathrm{I}}(k=1, \ldots, 4)$ are determined.

5. The linear algebraic system (8) is solved, and, thus, $\varphi_{k i}^{I}$ is obtained. Furthermore, $\varphi_{i}^{I}$ is also calculated from (7).

6. Using the Lagrange interpolation formula through five points, $\mathbf{V}_{\tau \mathrm{i}}^{\mathrm{I}}$ and $\mathrm{V}_{\mathrm{ni}}^{\mathrm{I}}$ are calculated. Next, $\mathrm{V}_{\mathrm{i}}^{\mathrm{I}}$ is determined from (10), and $\zeta_{i}^{I}$ is obtained from (6).

7. We proceed similarly at the next iteration step $n=2,3, \ldots$.

\section{CONCLUSIONS}

We address algorithmic aspects from the study of the compressible fluid's stationary motion through profile grids. The distinctive features of our framework consist in (i) using the boundary element method with real values; (ii) applying the successive approximation method for solving the integral equation of the velocity potential; and (iii) using the Lagrange interpolation formula through five points for calculating the derivatives of the velocity potential. Future work includes theoretical and practical improvements of our method.

\section{ACKNOWLEDGEMENTS}

The second author is supported by a Hertha Firnberg Research grant (T425-N23) of FWF (Austrian Science Fund).

\section{REFERENCES}

Hromadka II., T. V. (1984) The Complex Variable Boundary Element Method, Springer, ISBN 3-540-13743-2, Berlin

Kovacs, A. (2005) Classical and Modern Mathematical Methods in the Hydrodynamics of Profile Grids (in German), Ed. Politehnica, ISBN 973-625-230-2, Timisoara

Kovacs, A. (1999) Stabilization and Determination of an Iteration Formula for a Convex Infinite Area (in German), Proc. of the 8th Symp. of Mathematics and its Applications, Romania, ISSN 1224-6069, Boja, N. et al. (Eds.), pp. 88-92, Ed. Politehnica, Timisoara

Kovacs A. \& Kovacs L. (2003) The Calculus Algorithm for the Integral Equation of the Compressible Fluids Velocity Potentials through Profile Grids (in German), Proc. of the 10th Symp. of Mathematics and its Applications, Romania, ISSN 1224-6069, Boja, N. et al. (Eds.), pp. 427-434, Ed. Politehnica, Timisoara

Kovacs, A. \& Kovacs, L. (2006) Using the Lagrange Interpolation Polynomial in Determining the Fluid's Complex Velocity through Profile Grids, Proc. of the 11th Symp.of Mathematics and its Applications, Romania, ISSN 1224-6069, Boja, N. et al. (Eds.), pp. 167-173, Ed. Politehnica, Timisoara 